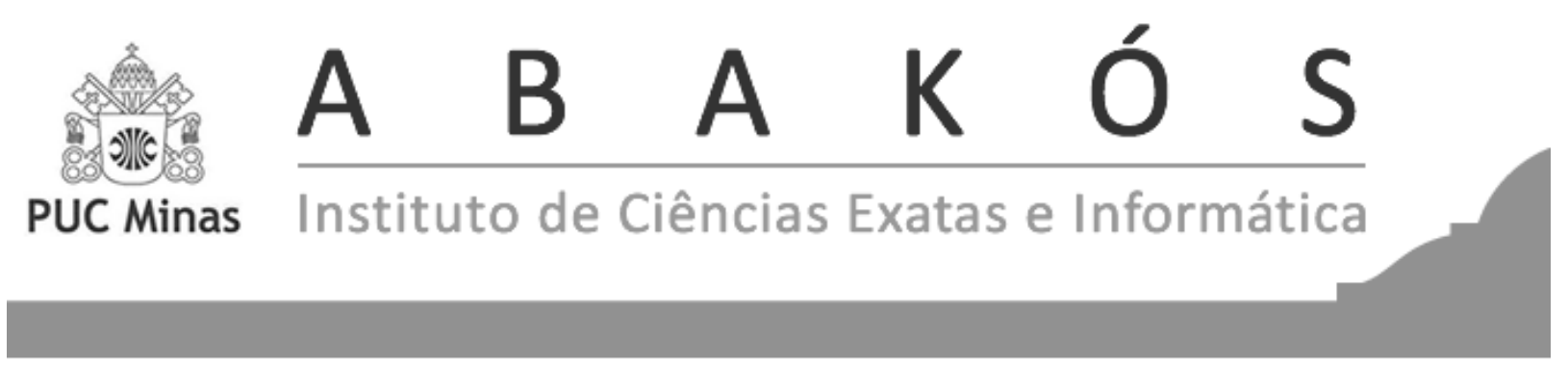

\title{
Aprendizado de Máquina Fuzzy Incremental para Classificação de Faltas em Sistemas de Potência*
}

Incremental Fuzzy Machine Learning for Power Systems Fault Classification

\author{
Márcio Wladimir Santana ${ }^{1}$ \\ Daniel Furtado Leite ${ }^{2}$
}

\begin{abstract}
Resumo
O conceito de Qualidade da Energia está relacionado a um conjunto de alterações que podem ocorrer no sistema elétrico. Tais alterações (distúrbios/faltas) podem ocorrer em várias partes do sistema de energia, sejam nas instalações elétricas dos consumidores ou no sistema supridor da concessionária, causando prejuízos financeiros a ambas as partes. Por isso, é de fundamental importância a detecção e classificação em tempo real desses distúrbios de modo automático. Para reconhecimento e classificação de padrões, são considerados modelos inteligentes evolutivos, ou seja, modelos equipados com algoritmos de aprendizado incrementais online capazes de alterar seus parâmetros e estrutura, conforme novas informações surgem em um fluxo de dados. Em particular, é considerada a modelagem evolutiva baseada em conjuntos fuzzy (FBeM). Para o pré-processamento dos dados mensurados, e extração de variáveis indicadoras da presença de distúrbios, é considerado o filtro Hodrick-Prescott, a técnica de transformada rápida de Fourier e o valor eficaz da tensão. O modelo desenvolvido neste trabalho tem alcançado um desempenho comparável àqueles de modelos estado da arte na área de qualidade de energia. Detecção e classificação de distúrbios tais como elevação de tensão, sub-harmônico, transitório oscilatório, spikes e notching, ocorrendo de forma simultânea ou não, são alcançadas com acurácia de, aproximadamente, $99 \%$.
\end{abstract}

Palavras-chave: Qualidade de energia. Classificação de distúrbios. Aprendizado incremental on-line. Sistemas fuzzy evolutivos.

\footnotetext{
*submetido em 01/09/2018 e aceito em 07/08/2020

${ }^{1}$ Mestre em Engenharia de Sistemas e Automação pela Universidade Federal de Lavras - UFLA. Professor no Centro Federal de Educação Tecnológica de Minas Gerais - CEFET-MG Campus Nepomuceno, Brasil- marciosantana@cefetmg.br

${ }^{2}$ Doutor pela Faculdade de Engenharia Elétrica e de Computação da Universidade Estadual de Campinas (FEECUNICAMP). Professor do Departamento de Automática da Universidade Federal de Lavras (DAT-UFLA), Brasildaniel.leite@ufla.br
} 


\begin{abstract}
The concept of power quality is related to a set of changes that may occur in the electrical system.These result in flaws or bad consumer equipment operation. Such changes (disturbances/faults) can occur in many parts of a power system, including the consumer installation and the supplying system, which may yield financial losses to both. Real-time automatic detection and classification of disturbances are therefore of fundamental importance. In this study, evolving intelligent models, that is, models supplied with incremental online learning algorithms capable of changing their parameters and structure according to new information that emerges from a data stream, are considered for pattern recognition and classification. In particular, an evolving fuzzy set-based model (FBeM) is taken into consideration. A Hodrick-Prescott filter combined with a Fast Fourier Transform technique and root mean square voltages are considered for preprocessing measured data and extracting features that indicate the presence of disturbances. The model developed in this study has reached classification performance comparable to that of state-of-the-art models in the field of power quality. Detection and classification of disturbances such as voltage swell, sub-harmonic, oscillatory transient, spikes, and notching, occurring simultaneously, were reached with an accuracy of about $99 \%$.
\end{abstract}

Keywords: Power quality. Classification of disturbances. Incremental online learning. Evolving fuzzy systems. 


\section{INTRODUÇÃO}

Conforme os benefícios da energia elétrica passam a fazer parte do dia a dia das pessoas, é natural que se inicie um processo de discussão quanto à qualidade da energia elétrica (QEE) (DUGAN, 2003; OLIVEIRA, 2000; FERREIRA, 2010). Segundo Oliveira (2000), numa análise inicial preocupa-se com a continuidade do serviço, já que qualquer falha no fornecimento pode causar grandes prejuízos financeiros e pessoais. A questão da QEE surge a partir do momento em que os consumidores constatam pequenas interrupções no fornecimento. À medida que tais consumidores tornam-se mais sofisticados sob o ponto de vista tecnológico, outros fatores começam a ser considerados. Isso se justifica, principalmente, pelos seguintes motivos: os equipamentos utilizados nos dias de hoje são mais sensíveis a variações no sinal elétrico; o crescente interesse pela racionalização da energia elétrica tem aumentado o uso de equipamentos eletrônicos que aumentam os níveis de distorções harmônicas; maior conscientização dos consumidores em relação aos fenômenos ligados à qualidade da energia; integração de processos industriais, e o crescente uso de geração distribuída e de fontes alternativas de geração de energia.

Diante do exposto, é evidente a importância de análises e sistemas de monitoramento da QEE objetivando determinar as causas e as consequências dos distúrbios no sistema. Este trabalho tem motivações em nível de método e em nível de aplicação. A motivação em se adotar um método de filtragem e decomposição para extração de variáveis, em combinação com modelo fuzzy munido de algoritmos incrementais online é devido ao fato que essas abordagens têm se mostrado eficientes para tratar problemas de detecção e classificação de anomalias em diferentes contextos. Por exemplo, elas têm sido consideradas para detecção e classificação de novidades em séries temporais econométricas e climáticas; detecção de faltas em máquinas elétricas; detecção de mudanças abruptas e graduais em aplicações biomédicas, entre outras (GARCIA et al., 2019; LEITE; GOMIDE, 2012; LEITE et al., 2016; LEITE; ŠKRJANC, 2019; ŠKRJANC et al., 2019)

Algoritmos de detecção e classificação de distúrbios devem ser aptos a lidar com a ocorrência de novidades nos padrões dos dados. Novidades devem ser detectadas e armazenadas em modelos auto ajustáveis em modo online (GARCIA et al., 2019; SILVA et al., 2018; LEITE et al., 2009; SOARES et al., 2019). A quantidade de dados e amostras é grande. Dessa forma, por vezes, é inviável armazená-los em bases de dados para posteriormente empregar algoritmos e modelos convencionais, isto é, algoritmos baseados em análises estatísticas convencionais ou modelos inteligentes estáticos, pré-treinados em modo offline, como as redes neurais artificiais. Outra questão que dificulta o desenvolvimento de sistemas de monitoramento da qualidade de energia baseados em métodos estatísticos e métodos convencionais de inteligência computacional é o surgimento de condições de falta/distúrbios não previstas. 
Atualmente é importante considerar o aumento das smart grids em nosso sistema elétrico. As smart grids são uma nova arquitetura de distribuição de energia elétrica que integra e possibilita ações a todos os usuários a ela conectados (EL-HAWARY, 2014; DI SANTO et al., 2015; BISOI et al., 2020). A partir desse novo conceito de geração e consumo de energia elétrica podem surgir distúrbios de QEE não previstos. Modelos de detecção e classificação fuzzy evolutivos são capazes de detectar novos distúrbios, pois sua base de regras evolui de acordo com o fluxo de dados.

Portanto, as contribuições desse trabalho são claras: detectar e classificar distúrbios no sistema elétrico de potência em tempo real. Esse processo deve acontecer em modo online, devendo apresentar boa precisão e baixo custo computacional. Foi considerada a modelagem evolutiva baseada em conjuntos fuzzy (FBeM - Fuzzy Set Based evolving Modeling) (LEITE; GOMIDE, 2012), que é novidade como método aplicado à análise da qualidade da energia elétrica. São abordados os seguintes distúrbios neste trabalho: sipke, notch, sub-harmônicos, transitório oscilatório e elevação de tensão.

\section{MATERIAL E MÉTODOS}

Esta seção apresenta um método híbrido para obtenção de variáveis indicadoras de distúrbios, composto pelo filtro Hodrick-Prescott (HP), Transformada Rápida de Fourier e valor eficaz da tensão $\left(v_{R M S}\right)$.

\subsection{Filtro Hodrick-Prescott}

O filtro HP, que é novidade do ponto de vista de técnicas para extração de características de QEE, decompõe o sinal em suas componentes de tendência e cíclica/aleatória. Em essência, removem-se as flutuações de baixa frequência dos sinais ou séries. A hipótese é que, nessa separação, a variabilidade de baixa frequência representa a tendência de prazo mais longo, e a variabilidade de frequência mais alta representa os ciclos mais curtos e/ou aleatórios (HODRICK; PRESCOTT, 1997). O filtro HP busca extrair a tendência, que é considerada estocástica, mas com variações suaves ao longo do tempo e não correlacionadas com o ciclo, através da minimização com respeito a $\tau_{t}$ da seguinte expressão:

$$
j=\min \sum_{t=1}^{T} \mathrm{C}_{2}^{t}+\lambda \sum_{t=3}^{T}\left(\Delta^{2} \tau_{t}\right)^{2},
$$

onde $c_{t} \triangleq y_{t}-\tau_{t}$ e $\Delta^{2}=(1-L)^{2}$, com L sendo o operador de defasagens, por exemplo $L x_{t}=x_{t-1}$.

Na Equação 1, $T$ é a quantidade de amostras de dados em questão, e $\lambda$ é um parâmetro que penaliza a variabilidade da tendência. O parâmetro $\lambda$ é o parâmetro de suavidade com 
o qual se controla a aceleração da componente de tendência, isso é, as variações na taxa de crescimento da componente de tendência. O primeiro termo da Equação 1 é a soma dos desvios da série com respeito à tendência ao quadrado, e é uma medida do grau de ajuste. O segundo termo é a soma de quadrados das segundas diferenças das componentes de tendência e é uma medida do grau de suavidade.

\subsection{Transformada Rápida de Fourier}

A Transformada de Fourier é uma das ferramentas mais utilizadas na análise de sinais no domínio da frequência. A ideia é que qualquer sinal periódico pode ser descrito por um somatório de senos e cossenos (LATHI; GREEN, 2005). Existem duas abordagens para análise do espectro de frequências de um sinal: (i) a análise é feita de posse de uma função contínua; e (ii) somente se dispõe de dados mensurados, isso é, dispõe-se de uma função discreta. para estes casos, pode-se utilizar a Transformada de Fourier Contínua e Discreta, respectivamente. As relações representam as definições da Transformada Contínua e sua inversa; e concernem às definições da Transformada Discreta e sua inversa.

$$
\begin{gathered}
F(j \omega)=\int_{0}^{\infty} f(t) e^{-j \omega t} d t, \\
F(j \omega)=\frac{1}{2 \pi} \int_{-\infty}^{\infty} F(j \omega) e^{j \omega t} d \omega, \\
T F D\left(f_{n}\right)=\frac{1}{N} \sum_{k=0}^{N-1} x_{k} e^{-j 2 \pi f_{n} k \Delta t}, \\
x_{k}=\frac{1}{\Delta t} \sum_{f_{n}=0}^{(1-N) / T} T F D\left(f_{n}\right) e^{j 2 \pi f_{n} k \Delta t},
\end{gathered}
$$

onde $\omega$ é a frequência angular; $x_{k}$ é o sinal discreto; $T$ é o intervalo de tempo total medido; $\Delta T$ corresponde aos intervalos discretos de tempo; $N=T / \Delta T$ é o número de amostras; e $f_{n}=n / T, n=0,1,2, \ldots, N-1$, são as componentes discretas de frequência.

\subsection{Raiz do Valor Quadrático Médio}

O valor RMS (root mean square) ou valor eficaz é uma medida da magnitude de uma quantidade variável. O valor pode ser calculado para uma série de valores discretos ou para uma função contínua. $\mathrm{O}$ valor eficaz da tensão de um sinal elétrico é um atributo eficiente para detecção de distúrbios, em especial daqueles que geram variações consideráveis na amplitude do sinal, como afundamento/elevação de tensão, e interrupções. O valor eficaz de um sinal $x$ para uma coleção de $N$ valores $\left\{x_{1}, x_{2}, \ldots, x_{N}\right\}$ é dado por: 


$$
x_{R M S}=\sqrt{\frac{1}{N} \sum_{i=1}^{N} x(i)^{2}},
$$

onde $N$ é o número de amostras.

Para uma função variável contínua, $f_{(t)}$, definida no intervalo $T_{1} \leq t \leq T_{2}$, o valor RMS de um sinal $x$ é dado por:

$$
x_{R M S}=\sqrt{\frac{1}{T_{2}-T_{1}} \int_{T_{1}}^{T_{2}}[f(t)]^{2} d t} .
$$

\section{MODELAGEM EVOLUTIVA BASEADA EM CONJUNTOS FUZZY}

Modelagem evolutiva baseada em conjuntos fuzzy (FBeM, Fuzzy set Based evolving Modeling) emprega grânulos de informação tipo fuzzy, para construir mapas granulares que de maneira geral associam dados granulares de entrada a dados granulares de saída. Grânulos fuzzy garantem a generalidade da estrutura dos dados e proveem algoritmos com matemática simples e regras linguísticas descrevendo seu comportamento. Basicamente, um sistema FBeM pode perceber os dados de um fluxo sob diferentes resoluções, e decidir entre adotar granularidades mais simples ou mais detalhadas (LEITE; GOMIDE, 2012).

Modelos FBeM são criados e evoluídos quando requisitados pelo fluxo de dados. O algoritmo de aprendizagem de FBeM cria, expande e retrai grânulos recursivamente. Eventualmente, a estrutura granular resultante pode ser melhorada de acordo com relações intergranulares. FBeM combina sistemas fuzzy linguísticos e funcionais para prover aproximações singulares e granulares de funções não estacionárias. Sistemas fuzzy funcionais são, geralmente, mais precisos enquanto que os sistemas fuzzy linguísticos são mais interpretáveis. Através da combinação destes, FBeM aproveita as vantagens de ambos os sistemas simultaneamente. Em nível prático, especialistas preferem que sistemas online deem resultados aproximados tão bem quanto limites de tolerância nas aproximações (LEITE; GOMIDE, 2012).

\subsection{Modelagem Fuzzy Evolutiva}

FBeM é uma abordagem de modelagem incremental a partir de dados detalhados e um algoritmo de aprendizagem. Sua resposta global sucede da agregação de respostas locais mais específicas. O algoritmo incremental de FBeM molda sua estrutura de regras para aceitar novidades, lidar com incerteza, prover aproximações singulares e granulares de funções. FBeM lida com questões como bases de dados ilimitadas e escalabilidade (LEITE; GOMIDE, 2012). Modelos FBeM consistem de regras obtidas a partir de um sistema complexo. A aprendizagem em FBeM não requer pré-concepção de regras. Regras são criadas e adaptadas dinamicamente, consoante com o comportamento da função do processo ou da fronteira de decisão ao longo do 
tempo. Sempre que amostras de dados são disponibilizadas, um mecanismo de decisão pode acrescentar novas regras à estrutura FBeM ou adaptar parâmetros de regras existentes. Especialistas podem desejar prover uma descrição verbal sobre o processo a partir da intuição e experiência (LEITE; GOMIDE, 2012). Em modelos FBeM, regras $R^{i}$ governando grânulos de informação $y^{i}$ são do tipo:

$$
\begin{gathered}
S E\left(x_{1} \text { é } A_{1}^{i}\right) E \ldots E\left(x_{j} \dot{e} A_{j}^{i}\right) E \ldots E\left(x_{n} \text { é } A_{n}^{i}\right), E N T \tilde{A} O\left(y_{1} e^{\prime} B_{1}^{i}\right) E^{\prime} y_{1}=p_{1}^{i}\left(x_{j} \forall j\right) E \ldots \\
\ldots E\left(y_{k} e^{\prime} B_{k}^{i}\right) E^{\prime} y_{k}=p_{k}^{i}\left(x_{j} \forall j\right) E \ldots E\left(y_{m} \dot{e} B_{m}^{i}\right) E^{\prime} y_{m}=p_{m}^{i}\left(x_{j} \forall j\right)
\end{gathered}
$$

onde $x_{j}$ e $y_{k}$ são variáveis do fluxo de dados $(x, y)^{[h]}, h=1, \ldots ; A_{j}^{i}$ e $B_{k}^{i}$ são funções de pertinência construídas a partir dos dados disponibilizados; e $p_{k}^{i}$ são polinômios de aproximação.

As regras $R^{i}, i=1, \ldots, c$, formam a base. Note que uma regra FBeM combina consequentes linguístico e funcional. O consequente linguístico envolve funções e provê interpretabilidade aos resultados. O consequente funcional oferece aproximação singular e precisão. Com essa estrutura, FBeM toma vantagem de ambos, sistemas linguísticos e funcionais, em uma plataforma de modelagem única. Um aspecto a ser levado em conta em granulação tipo espalhamento refere-se a busca por uma quantidade factível de partições, posições e tamanhos de grânulos. Essencialmente, FBeM emprega funções de pertinência Gaussianas como objetos granulares formais para envolver a incerteza dos dados (LEITE; GOMIDE, 2012). Conforme Leite e Gomide (2012), um conjunto fuzzy Gaussiano $A_{j}^{i}=G\left(\mu_{j}^{i}, \sigma_{j}^{i}\right)$ é caracterizado pelo valor modal $\mu_{j}^{i}$ e espalhamento $\sigma_{j}^{i}$. Características que fazem essa representação apropriada incluem: (i) facilidade de aquisição dos parâmetros. O valor modal e de espalhamento são capturados diretamente a partir do fluxo de dados; (ii) suporte infinito. Visto que o domínio dos dados é desconhecido anteriormente ao aprendizado, o suporte de Gaussianas estende-se ao longo de todo o domínio; (iii) suavidade e superfície continuamente diferenciável. O consequente de regras FBeM inclui funções locais afins do tipo:

$$
p_{k}^{i}=a_{0 k}^{1}+\sum_{j=1}^{n} a_{j k}^{i} x_{j}
$$

Em geral, funções $p_{k}^{i}$ podem ser de tipos diferentes e não requerem linearidade. $\mathrm{O}$ algoritmo Mínimos Quadrados Recursivo (RLS) é usado para determinar os coeficientes locais $a_{j k}^{i}$. A representação Gaussiana permite sobreposição de todos os grânulos. Consequentemente, cada regra FBeM contribui à saída do sistema. A saída singular de FBeM é determinada como um valor médio ponderado sobre todas as regras:

$$
p_{k}=\frac{\sum_{i=1}^{c} \min \left(A_{1}^{i}, \ldots, A_{n}^{i}\right) p_{k} i}{\sum_{i=1}^{c} \min \left(A_{1}^{i}, \ldots, A_{n}^{i}\right)}
$$

isso assegura transição suave entre funções de pertinência.

De forma similar a abordagem para agrupar dados em conjuntos antecedentes $A_{j}^{i}$, conjuntos consequentes $B_{k}^{i}$ usam granulação tipo espalhamento, por exemplo, funções Gaussianas $B_{k}^{i}=G\left(\mu_{k}^{i}, \sigma_{k}^{i}\right)$. A saída granular $B_{k}^{i}$ provê informação mais importante que a saída numérica 
$P_{k}$. Não obstante, em problemas de classificação, o termo consequente de uma regra FBeM é essencialmente uma classe (LEITE; GOMIDE, 2012).

\subsection{Aprendizagem Recursiva On-line}

FBeM aprende a partir de um fluxo $(x, y)^{[h]}, h=1, \ldots$, onde $y^{[h]}$ é conhecido dado $x^{[h]}$ ou se tornará conhecido alguns passos adiante. Cada par $(x, y)$ é uma observação da função $f$. Quando $f$ muda com o tempo, dizemos que a função é não estacionária. O procedimento de aprendizagem para evoluir modelos FBeM é sumarizado, como se segue, no Algoritmo 1.

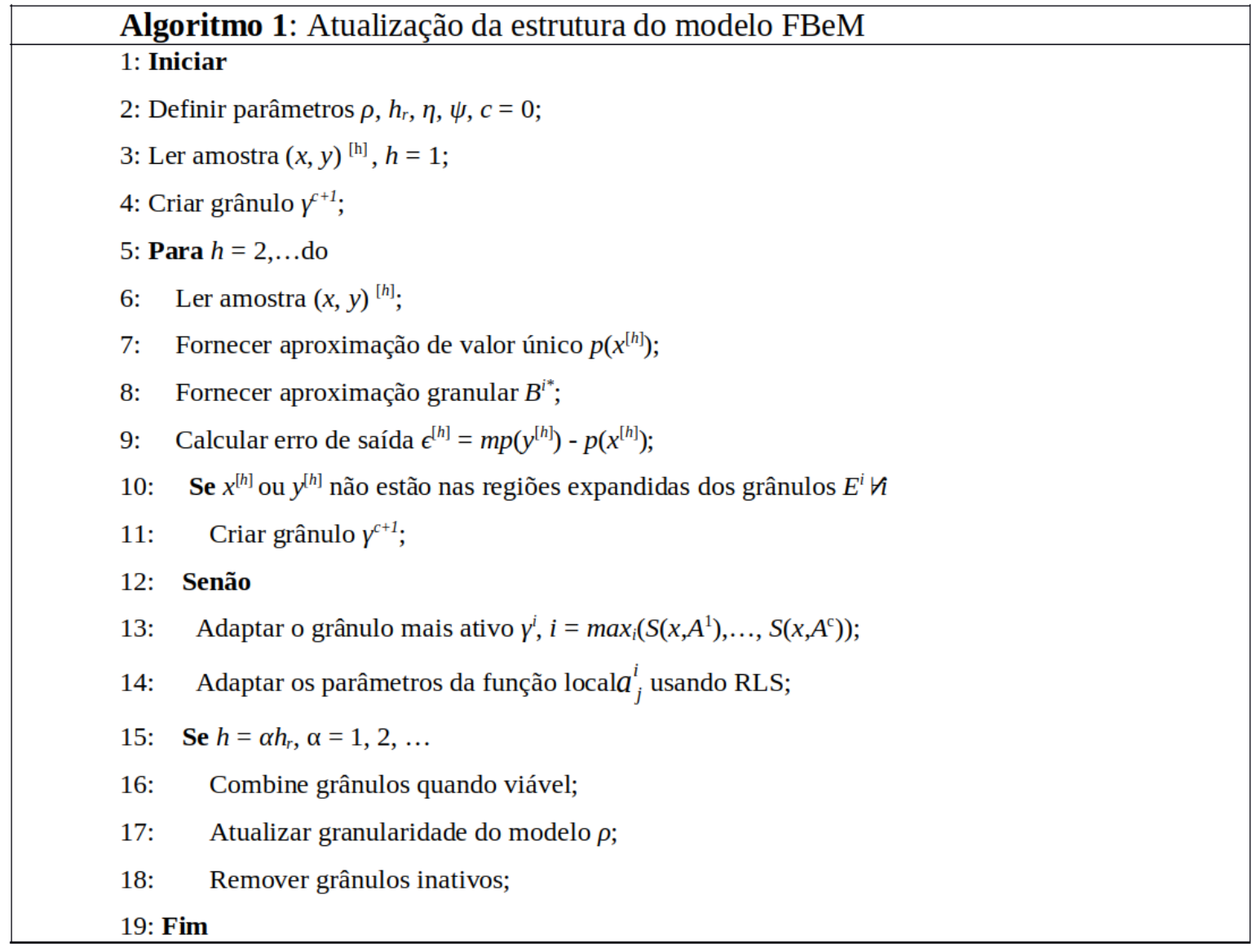

Os passos 2 e 18 enfatizam a essência de algoritmos dirigidos a fluxos, isso é, amostras são lidas e descartadas uma por vez. Dados históricos são descartados. Modelos granulares evoluem sempre que novas informações surgem nos dados, passo 6. Quando uma nova amostra não condiz com o conhecimento atual, o procedimento cria um grânulo e uma regra, passo 11 . Ao contrário, se uma nova amostra ajusta-se ao conhecimento atual, o procedimento adapta grânulos e regras existentes, passo 14. Eventualmente, a estrutura resultante poderá ser otimizada de acordo com relações intergranulares, passo 17. 


\subsection{Criação de Regras}

Em FBeM, regras não existem a priori. Elas são criadas e evoluem a medida que os dados são disponibilizados. Um novo grânulo $\gamma^{c+1}$ e a $R^{c+1}$ regra que o governa são criados quando as regras existentes não são suficientemente ativadas para uma amostra $x^{[h]}$. FBeM assume que a amostra traz uma nova informação sobre o processo. Seja, $\rho \epsilon[0,1]$ um limiar que determina quando criar ou adaptar regras (LEITE; GOMIDE, 2012). Se

$$
\min \left(A_{1}^{i}, \ldots, A_{n}^{i}\right) \leq \rho \forall j
$$

então a estrutura FBeM é expandida.

Note que se $\rho$ é 0 , então o sistema é estruturalmente estável e incapaz de capturar mudanças de conceito. Ao contrário, se $\rho$ é 1, FBeM cria uma regra para cada nova amostra, o que não é prático. Adaptabilidade é alcançada em situações intermediárias (compromisso estabilidade/plasticidade) (LEITE; GOMIDE, 2012).

O papel de $\rho$ é fundamental na determinação da granularidade de modelos FBeM. Escolhas de $\rho$ impactam na precisão e transparência de modelos, resultando em diferentes visões granuladas e níveis de detalhes do mesmo processo. Um novo grânulo $\gamma^{c+1}$ é inicialmente representado por funções de pertinência, $A_{j}^{c+1}$ e $B_{k}^{c+1}$, com parâmetros:

$$
\begin{gathered}
\mu_{j}^{c+1}=x_{j}^{[h]}, \\
\mu_{k}^{c+1}=y_{k}^{[h]}, \\
\sigma_{j}^{c+1}=\sigma_{k}^{c+1}=1 / 2 \pi,
\end{gathered}
$$

Essa é a abordagem de Stiegler para funções Gaussianas padrões (STIEGLER, 1982). Os coeficientes de polinômios $p_{k}^{c+1}$ locais são:

$$
\begin{gathered}
a_{0 k}^{c+1}=y_{k}^{[h]} \\
a_{j k}^{c+1}=0, j \neq 0
\end{gathered}
$$

Com essa parametrização inicial a preferência é dada ao projeto de grânulos balanceados ao longo de suas dimensões ao invés de grânulos com geometria desbalanceada. Consequentemente, FBeM implementa o princípio da granularidade justificada por Wang et al. (2018) e tende a desenvolver conjuntos fuzzy mais específicos no sentido de Yager (2008). 


\subsection{Adaptação de Regras}

Conforme Leite e Gomide (2012) a adaptação de regras consiste em (i) expandir ou contrair $A_{j}^{i}$ e $B_{k}^{i}$ para acomodar novos dados; (ii) mover grânulos $\gamma^{i}$ na direção de regiões de dados mais densas; e (iii) ajustar os coeficientes de funções locais $p_{k}^{i}$. Uma regra $R^{i}$ é adaptada sempre que é suficientemente ativada por uma amostra $x^{[h]}$ de acordo com:

$$
\min \left(A_{1}^{i}, \ldots, A_{n}^{i}\right) \leq \rho
$$

Geometricamente, a amostra pertence a uma região influenciada pelo grânulo $\gamma^{i}$. Para incluir $x^{[h]}$, FBeM atualiza o valor modal e o espalhamento das funções de pertinência $A_{j}^{i}$ correspondentes como segue:

$$
\begin{gathered}
\mu_{j}^{i}(\text { novo })=\frac{(\varpi-1) \mu_{j}^{i}(\text { velho })+x_{j}}{\varpi^{i}}, \\
\sigma_{j}^{i}(\text { novo })=\frac{\left(\varpi^{i}-1\right)}{\varpi^{i}} \sigma_{j}^{i}(\text { velho })+\frac{1}{\left(\varpi^{1}-1\right)} x_{j}-\left(\mu_{j}^{i}(\text { novo })\right)^{2},
\end{gathered}
$$

onde $\varpi^{i}$ refere-se ao número de vezes que o i-ésimo grânulo foi ativado pelo fluxo de dados.

Nota-se que os valores são calculados recursivamente e, portanto, não demandam acumulação de dados. Apenas a regra mais ativa para $x^{[h]}$ é escolhida para adaptação. A adaptação de conjuntos fuzzy consequentes $B_{k}^{i}$ usa dados de saída $y_{k}^{[h]}$. Coeficientes polinomiais $a_{j k}^{i}$ são atualizados usando o algoritmo RLS (LEITE; GOMIDE, 2012).

\subsection{Ajuste da Granularidade}

O limiar $\rho$ assume valores no intervalo unitário de acordo com erros de predição. Níveis de ativação de regras para uma dada entrada $x^{[h]}$ são comparados com o valor de $\rho^{[h]}$ e definem mudança paramétrica ou estrutural de modelos FBeM. Valores de $\rho$ influenciam a granularidade e inteligibilidade de modelos. No caso mais geral, FBeM começa a aprender a partir de uma base de regras vazia, sem qualquer conhecimento sobre a propriedades dos dados. Consequentemente, é justo iniciar $\rho$ em uma condição intermediária para permitir estabilidade e plasticidade estrutural igualmente. Usa-se $\rho^{[o]}=0.5$ como valor padrão.

Seja E o erro quadrado máximo entre predições $p_{k}\left(x^{[h]}\right)$ e valores reais $y_{k}^{[h]}$, então:

$$
e_{k}=\left(y_{k}^{[h]}-p_{k}\left(x^{[h]}\right)\right)^{2} k=1, . ., m
$$

$\mathrm{e}$

$$
E=\max \left(e_{1}, \ldots, e_{k}, \ldots, e_{m}\right) .
$$

Admita como o erro máximo aceitável. Logo, $\rho$ aprende valores para si mesmo a partir 
de:

$$
\rho(\text { novo })=\rho(\text { velho })+\alpha\left(E_{D}-E\right)
$$

onde $\alpha$ é a taxa de aprendizagem.

Especialistas escolhem o valor de $E_{D}$ e podem desejar que ele seja zero. Valores muito pequenos de $E_{D}$ conduzem $\rho$ a zero e levam a sobre ajuste (overfitting) de modelos. A prática sugere abrir mão de certa precisão para alcançar um erro de aproximação aceitável, abstrações granulares úteis e compactação da base de dados em regras interpretáveis. A adaptação recursiva da granularidade alivia escolhas arbitrárias do quão rápido e quão frequente a estrutura dos dados muda (LEITE; GOMIDE, 2012).

\subsection{Compactação da Estrutura Resultante}

Segundo Leite e Gomide (2012), relacionamentos entre pares de grânulos podem ser fortes o suficiente para justificar a formação de um grânulo maior e mais abstrato que herda a essência e natureza de grânulos menores e mais detalhados. A análise de relações intergranulares requer uma métrica para medir a distância entre objetos fuzzy. A distância entre dois grânulos, $\gamma^{i 1}$ e $\gamma^{i 2}$, pode ser calculada da seguinte forma:

$$
D\left(\gamma^{i 1}, \gamma^{i 2}\right)=\frac{1}{n} \sum_{j=1}^{n}\left\|\mu_{j}^{i 1}-\mu_{j}^{i 2}\right\|^{2}+\sigma_{j}^{i 1}+\sigma_{j}^{i 2}-2 \sqrt{\sigma_{j}^{i 1} \sigma_{j}^{i 2}}
$$

Essa medida considera objetos Gaussianos e a especificidade da informação que, por vez, é inversamente proporcional ao espalhamento. FBeM combina grânulos que apresentam o menor valor de $D($.$) para qualquer par de grânulos da coleção atual e um critério de deci-$ são. A decisão pode ser baseada em valor limiar $\Delta$ ou julgamento especialista a respeito da conveniência da mescla destes grânulos.

Um novo grânulo $\gamma^{i}$, combinação de $\gamma^{i 1}$ e $\gamma^{i 2}$, é construído por funções de pertinência Gaussianas com valor modal:

$$
\mu_{j}^{i}=\frac{\frac{\sigma_{j}^{i 1}}{\sigma_{j}^{i 2}} \mu_{j}^{i 1}+\frac{\sigma_{j}^{i 2}}{\sigma_{j}^{i 1}} \mu_{j}^{i 2}}{\frac{\sigma_{j}^{i 1}}{\sigma_{j}^{i 2}}+\frac{\sigma_{j}^{i 1}}{\sigma_{j}^{i 2}}}, j=1, . ., n
$$

e espalhamento

$$
\sigma_{j}^{i}=\sigma_{j}^{i 1}+\sigma_{j}^{i 2}, j=1, \ldots, n,
$$

Essas são relações heurísticas que basicamente levam em conta a proporção de incerteza em cada grânulo para determinar a localização e tamanho do novo grânulo. As mesmas relações de combinação valem para variáveis de saída $k$. Os coeficientes dos novos polinômios locais são:

$$
a_{j k}^{i}=\frac{1}{2}\left(a_{j k}^{i 1}+a_{j k}^{i 2}\right), j=0, \ldots, n
$$


Naturalmente, combinação de grânulos reduz o número de regras e redundância (LEITE; GOMIDE, 2012).

\subsection{Remoção de Grânulos}

Um grânulo deve ser removido da estrutura de FBeM se ele parece ser inconsistente com o conceito atual. Estratégias comuns de remoção consideram (i) apagar grânulos criados a muitas iterações; (ii) excluir grânulos baseados em valores de erro local ou (iii) apagar grânulos inativos. Neste trabalho, optamos pela estratégia de remover grânulos inativos. Grânulos antigos ainda podem ser úteis no ambiente atual, enquanto que grânulos que geram erros podem ser revistos através do procedimento de adaptação de parâmetros.

Grânulos FBeM são excluídos quando se tornam inativos durante um número de iterações, $h_{r}$. Se a aplicação requer memorização de eventos raros ou se sazonalidades são esperadas, então pode ser o caso de não excluir grânulos. Remover os grânulos inativos periodicamente ajuda a manter a base de regras atualizada (LEITE; GOMIDE, 2012).

\section{METODOLOGIA}

\subsection{Banco de Dados}

Os sinais de tensão foram gerados de forma sintética e de acordo com a regulamentação do IEEE (THE INSTITUTE OF ELECTRICAL AND ELECTRONICS ENGINEERS, 2009). Todos os sinais gerados apresentam frequência fundamental igual a $60 \mathrm{~Hz}$. A frequência de amostragem utilizada foi de $15360 \mathrm{~Hz}$. Dessa forma, 256 amostras por ciclo são obtidas. Essa taxa de amostragem foi escolhida por ser suficiente para caracterizar a maioria dos distúrbios presentes nos sistemas elétricos (FERREIRA, 2010). Os sinais sintéticos, gerados computacionalmente pelo software MatLabß 2011 (uso acadêmico - individual) são contaminados por ruído branco gaussiano, com média nula. O ruído branco foi definido de modo que atenda a relação sinal-ruído (SNR - signal-to-noise ratio) desejada:

$$
S N R=20 \log \frac{A}{\sqrt{2 \sigma}}[d B],
$$

onde $A$ é a amplitude do sinal fundamental e $\sigma$ é o desvio padrão do ruído.

Na prática, sinais de tensão medidos nos sistemas elétricos possuem relação sinal-ruído entre 50dB e 70dB (TOMIC et al., 2007). Neste trabalho são utilizadas relações sinal-ruído $20 \mathrm{~dB}, 30 \mathrm{~dB}, 40 \mathrm{~dB}, 50 \mathrm{~dB}$ e $60 \mathrm{~dB}$, a fim de verificar o comportamento do modelo proposto com a presença de altos níveis de ruído no sinal a ser analisado. Foram gerados 12000 sinais para cada valor de SNR e para cada quantidade de ciclos da senoide (um, quatro e dez ciclos), dos 
quais: 2000 não possuem distúrbios; 2000 possuem distúrbio spike; 2000 possuem distúrbio notch; 2000 possuem distúrbio sub-harmônico; 2000 possuem distúrbio transitório oscilatório e 2000 possuem distúrbio elevação de tensão. As características de cada evento de QEE, como amplitude, fase, frequência, são atribuídas de maneira aleatória em intervalos com distribuição uniforme. As amplitudes adotadas neste trabalho são normalizadas em pu (por unidade), ou seja, a tensão de pico nominal tem valor unitário. A fase é atribuída aleatoriamente, dentro do intervalo $[-\pi ; \pi]$ com distribuição uniforme.

\subsection{Sistema de Monitoramento Proposto}

O monitoramento de distúrbios, em geral, compreende basicamente a obtenção de variáveis e a aplicação de um algoritmo que realize a detecção e classificação baseada nas variáveis escolhidas como indicadoras da existência de distúrbios. Neste trabalho a obtenção de variáveis indicadoras de distúrbios foi feita a partir dos resultados obtidos com o emprego do filtro HP, da FFT e do valor eficaz. A construção do modelo detector/classificador foi realizada em modo online a partir de um fluxo de dados. A construção e adaptação de detectores/classificadores em modo online é uma clara contribuição do trabalho à área de QEE.

O esquema geral do sistema de monitoramento proposto neste trabalho é ilustrado pelo diagrama em blocos da Figura 1. O sinal a ser processado pelo classificador é o vetor "Dados de entrada" resultante do pré-processamento do sinal com distúrbio através filtro HP, Transformada Rápida de Fourier e do valor RMS.

Figura 1 - Sistema fuzzy de monitoramento de sistemas de potência proposto

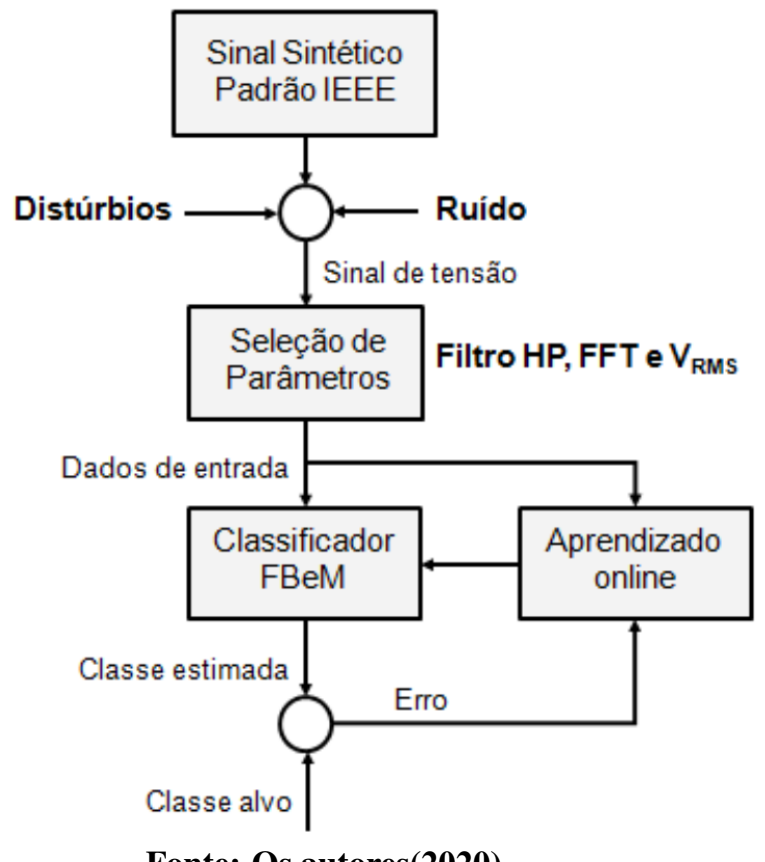

Fonte: Os autores(2020). 


\subsection{Aplicação do Filtro HP}

O filtro HP foi usado para decompor o sinal elétrico em suas componentes de tendência e cíclica na etapa de extração de parâmetros. Os parâmetros que foram extraídos são as amplitudes máximas e mínimas do sinal com distúrbio (antes do filtro) e da componente cíclica do filtro HP, que são os quatro primeiros parâmetros do vetor de entrada $\left(X_{1}, X_{2}, X_{3}\right.$ e $\left.X_{4}\right)$ no detector/classificador. A Figura 2 exemplifica um sinal elétrico típico. O sinal mostrado na Figura 2 tem frequência fundamental de $60 \mathrm{~Hz}$, amplitude de 1 pu e SNR de $30 \mathrm{~dB}$. Porém, o sinal está contaminado com o distúrbio transitório oscilatório proveniente do chaveamento de banco de capacitores.

Figura 2 - Exemplo de uso do Filtro HP aplicado sobre uma janela de tempo de 4 ciclos do sinal de tensão contendo distúrbio transitório oscilatório

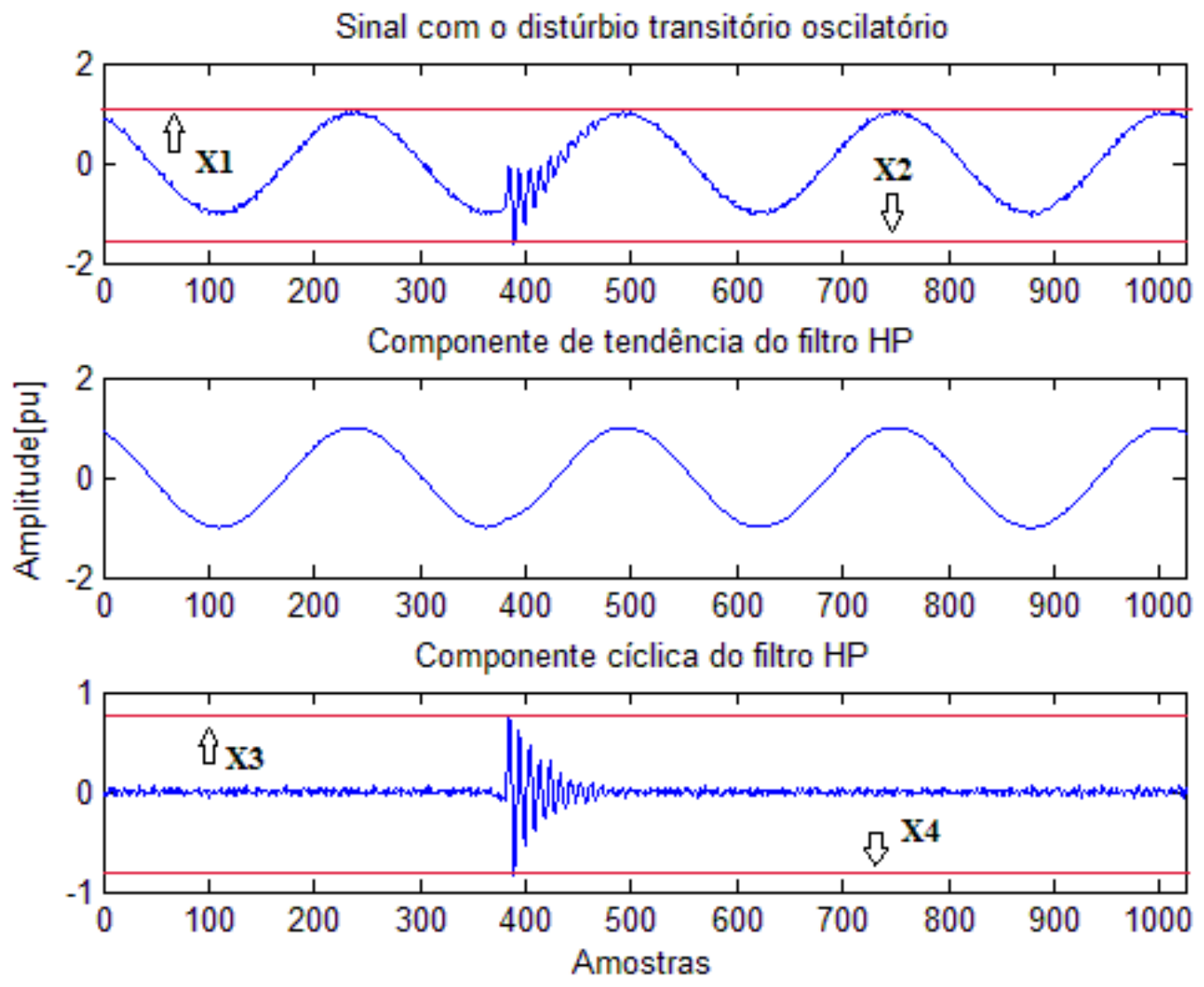

Fonte: Os autores (2020).

O filtro HP decompôs o sinal original em duas componentes, a se ver: componente de tendência e componente cíclica. O ruído presente no sinal, componente de alta frequência, apresentou-se na componente cíclica bem como o transitório oscilatório que também é uma componente de alta frequência. Na componente de tendência apresentou-se o sinal de frequência fundamental em $60 \mathrm{~Hz}$ sem distúrbio e sem ruído também.

Para comparar a aplicação do filtro HP considera-se o distúrbio spike nas mesmas condições de relação sinal-ruído e amplitude que o transitório oscilatório. Percebe-se na Figura 3, 
principalmente na componente cíclica do filtro HP, que é possível extrair padrões nos dados, ou seja, os parâmetros $\left(X_{3}\right.$ e $\left.X_{4}\right)$ são diferentes.

Figura 3 - Exemplo de uso do Filtro HP aplicado sobre uma janela de tempo de 4 ciclos do sinal de tensão contendo distúrbio do tipo spike
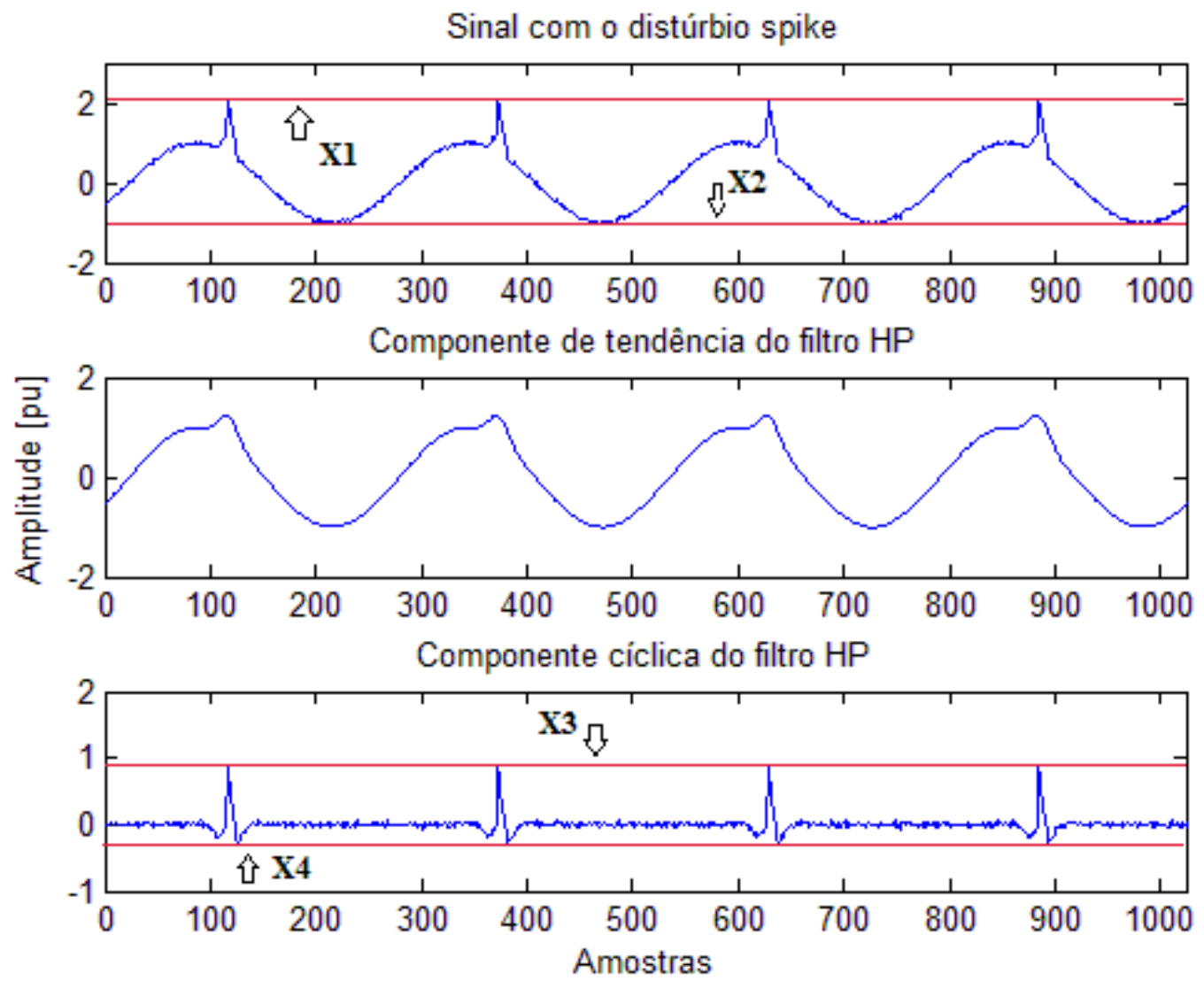

Fonte: Os autores (2020).

\subsection{Utilização da Transformada Rápida de Fourier}

A FFT foi utilizada para extrair o máximo valor do coeficiente da Transformada Discreta de Fourier para a frequência fundamental, que é o quinto parâmetro $\left(X_{5}\right)$ do vetor de entrada. Este coeficiente tem seu valor máximo alterado de acordo com alguns tipos de distúrbios, portando, justifica-se sua utilização como característica indicadora da presença de distúrbio, pois seu valor se altera dependo do distúrbio.

Para evidenciar a variação dos valores dos valores máximos do coeficiente da FFT na frequência fundamental do sistema utilizam-se as Figuras 4 e 5. Na Figura 4 tem-se o distúrbio sub-harmônico na frequência de $17 \mathrm{~Hz}$ e amplitude de $0.6 \mathrm{pu}$. Percebe-se que o valor do coeficiente da frequência fundamental da FFT do sistema é $1 \mathrm{pu}$.

Na Figura 5 tem-se um sinal com a presença do distúrbio elevação de tensão. Percebese que o valor do coeficiente da FFT para frequência fundamental é 1,12 pu. Dessa forma, consegue-se extrair características diferentes para diferentes classes de distúrbios. 
Figura 4 - Exemplo de espectro de Fourier extraído de um sinal de tensão contendo distúrbio do tipo sub-harmônico

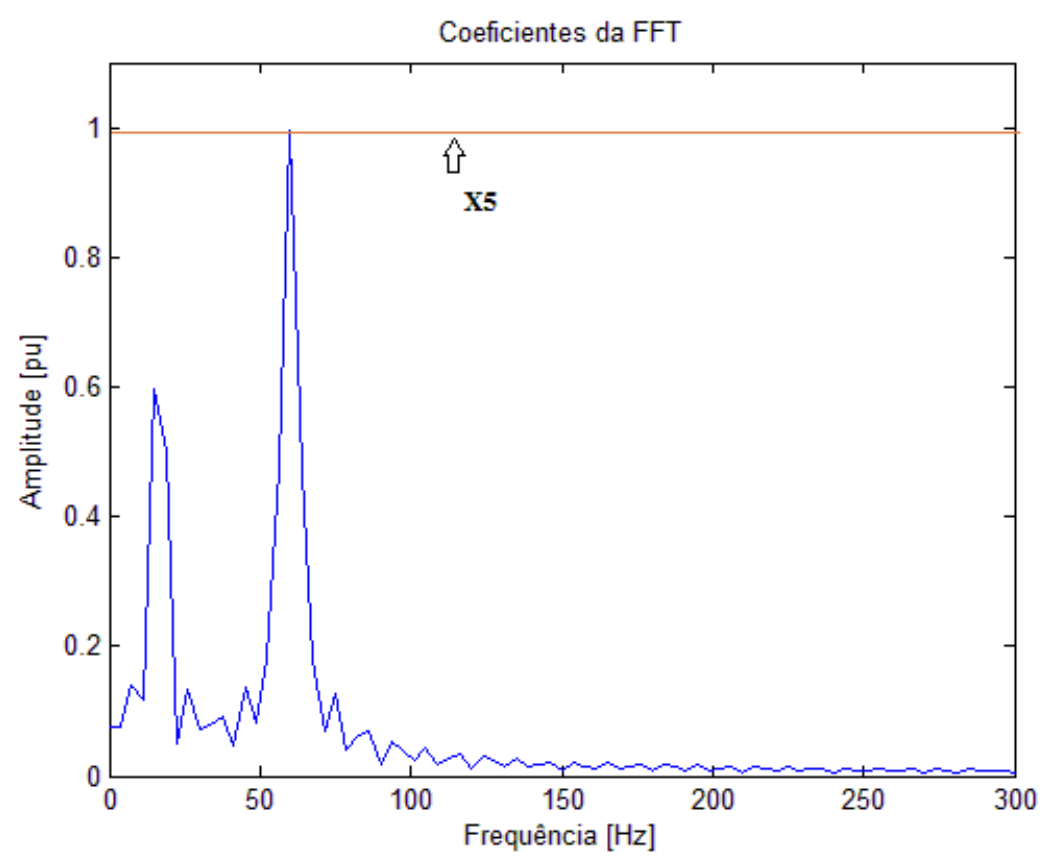

Fonte: Os autores (2020).

Figura 5 - Exemplo de espectro de Fourier extraído de um sinal de tensão contendo distúrbio do tipo elevação de tensão (voltage swell)

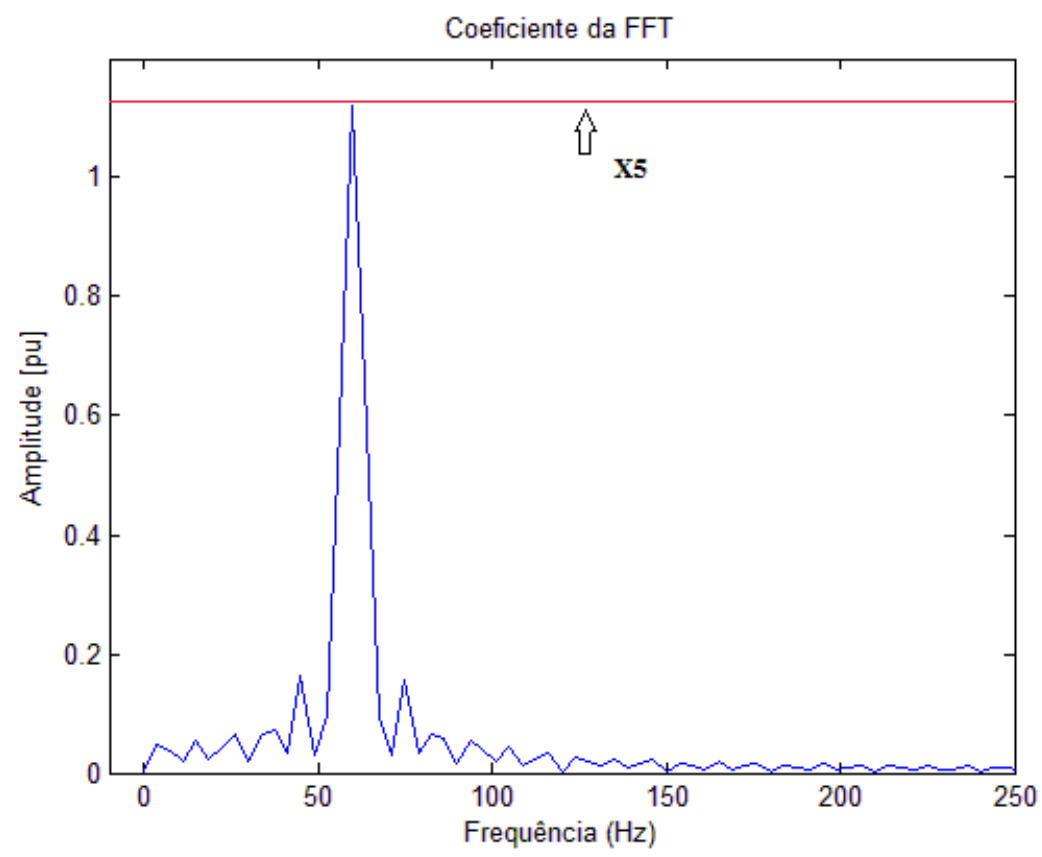

Fonte: Os autores (2020).

\subsection{Valor Eficaz da Tensão da Componente HP de Tendência}

Neste trabalho o valor eficaz da tensão da componente de tendência do filtro HP foi utilizado como um dos parâmetros analisados pelo detector/classificador proposto. É o sexto 
$\left(X_{6}\right)$, e último, parâmetro do vetor de entrada. Nos sinais com deformação na forma de onda o valor eficaz da tensão se altera também, justificando-se assim, sua aplicação. Na Figura 6 tem-se o distúrbio sub-harmônico de $20 \mathrm{~Hz}$, amplitude de 0,4 pu e valor eficaz da tensão da componente de tendência de 0,7664 pu. Para o sinal sem distúrbio de 1 pu de amplitude o valor eficaz é de 0,7071 pu. Nota-se, na Figura 6, que a componente de tendência está livre das componentes de alta frequência presentes no sinal analisado, ou seja, livre dos ruídos. Isto é de fundamental importância na análise que está sendo proposta, pois estamos considerando baixos valores de SNR.

Figura 6 - Exemplo de decomposição proporcionada pelo filtro HP para um sinal de tensão com relação sinal-ruído SNR de 20 dB
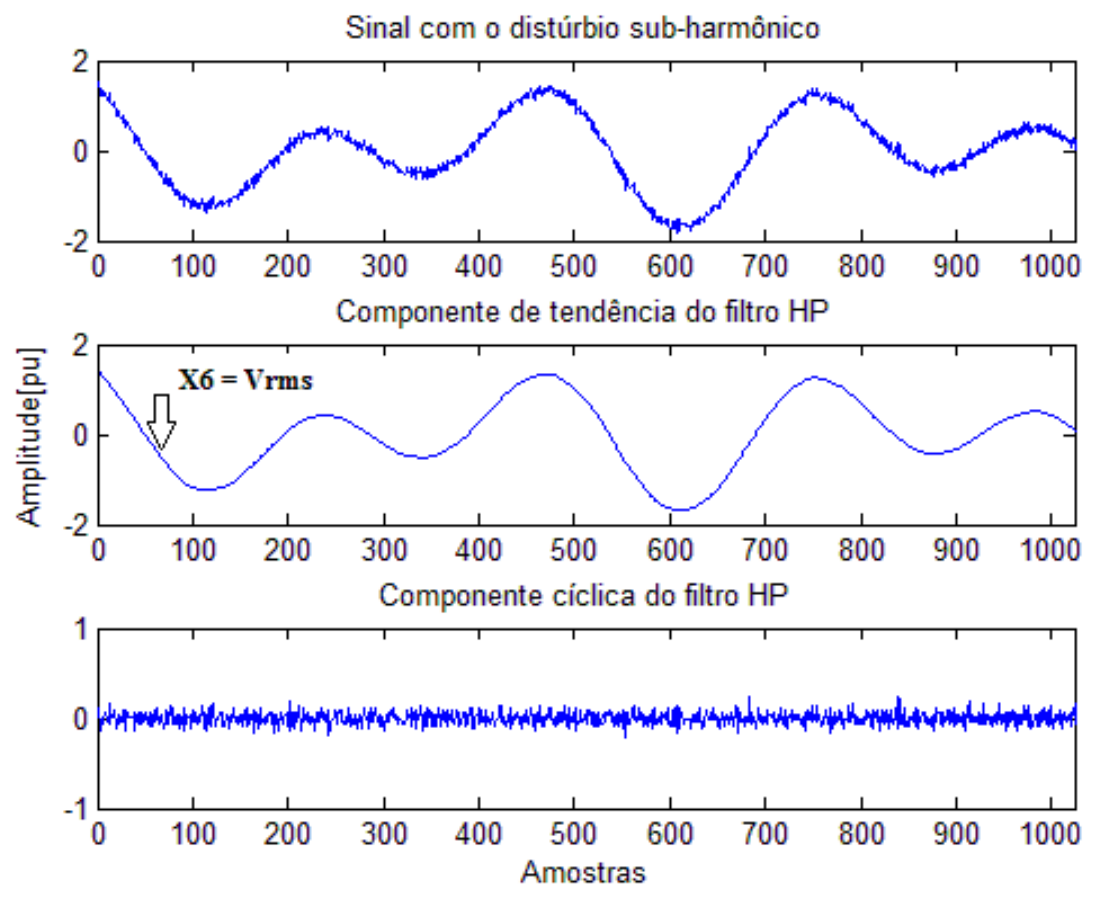

Fonte: Os autores (2020).

Portanto, o vetor de entrada do classificador é composto por seis parâmetros:

$X_{1}$ : valor máximo do sinal de tensão incluindo o distúrbio;

$X_{2}$ : valor mínimo do sinal de tensão incluindo o distúrbio;

$X_{3}$ : valor máximo da componente cíclica do filtro HP;

$X_{4}$ : valor mínimo da componente cíclica do filtro HP;

$X_{5}:$ amplitude do coeficiente da FFT na componente fundamental $60 \mathrm{~Hz}$;

$X_{6}$ : valor eficaz da componente de tendência do filtro HP. 


\section{RESULTADOS E DISCUSSÃO}

Neste item o sistema de detecção e classificação, apresentado na seção anterior, foi avaliado. Diferente da maioria dos trabalhos de detecção e classificação de distúrbios de QEE conhecidos na literatura, onde uma base de dados é conhecida a priori e os sistemas são implementados de forma offline (LIU et al., 2018; ZHU et al., 2019), o presente trabalho apresenta uma análise de forma online através de fluxo de dados em tempo real. Dessa forma, não há conhecimento a priori dos dados a serem analisados.

\subsection{Detecção de Distúrbios}

Neste item foi analisado o desempenho de detecção do modelo FBeM para verificar se o modelo consegue distinguir o sinal sem distúrbio do sinal com distúrbio. Foi simulado o fluxo de dados contendo apenas um distúrbio e o sinal sem distúrbio, ou seja, duas classes. Ressaltase que modelo proposto detecta e classifica os eventos ao mesmo tempo e de maneira recursiva, conforme apresentado no item 5.2 deste trabalho. O processo foi repetido para cada distúrbio considerado. Os sinais analisados contêm um, quatro e dez ciclos da senoide a fim de verificar a robustez do sistema a várias janelas de análise. Quanto menor a quantidade de ciclos de análise mais difícil é o problema. A relação sinal-ruído terá variação entre $20 \mathrm{~dB}$ a $60 \mathrm{~dB}$. A acurácia do modelo proposto é calculada por:

$$
A c c=\frac{V P+V N}{V P+V N+F P+F N} \cdot 100
$$

em que $V P$ e $V N$ são os verdadeiros positivos e negativos, respectivamente; $F P$ e $F N$ são os falsos positivos e negativos, respectivamente.

A Tabela 1 apresenta o desempenho ( $A c c$ ) de detecção para os distúrbios considerados em função da relação sinal-ruído e do número de ciclos da senoide. Percebe-se pela Tabela 1 que os melhores resultados foram para os distúrbios transitório oscilatório, notch, spike, evidenciando que o sistema de detecção e os parâmetros utilizados a partir do filtro HP, FFT e valor eficaz são eficientes, nesses casos, mesmo para baixos valores de relação sinal-ruído e independente do número de ciclos da senoide analisados. Para o distúrbio sub-harmônico o resultado ficou acima dos 99\% para SNR de $20 \mathrm{~dB}$ a $60 \mathrm{~dB}$ e dez ciclos de análise da senoide, nos indicando que o número baixo de ciclos de análise interfere de maneira negativa na detecção deste distúrbio. Para o distúrbio elevação de tensão nota-se que os melhores resultados foram para 10 e 4 ciclos de análise independente da SNR, mostrando a imunidade, nesse caso, ao ruído no sinal. 
Tabela 1 - Desempenho em detecção da condição do sistema de potência reportada pelo modelo proposto, FBeM

\begin{tabular}{c|c|c|c|c|c|c}
\hline Distúrbios & Ciclos & $20 \mathrm{~dB}$ & $30 \mathrm{~dB}$ & $40 \mathrm{~dB}$ & $50 \mathrm{~dB}$ & $60 \mathrm{~dB}$ \\
\hline \multirow{3}{*}{ Transitório oscilatório } & Um & 99,42 & 99,95 & 99,95 & 99,95 & 99,95 \\
\cline { 2 - 7 } & Quatro & 99,17 & 99,97 & 99,95 & 99,97 & 99,90 \\
\cline { 2 - 7 } & Dez & 98,72 & 99,92 & 99,92 & 99,92 & 99,95 \\
\hline \multirow{3}{*}{ Spike } & Um & 92,17 & 99,97 & 99,95 & 99,95 & 99,95 \\
\cline { 2 - 7 } & Quatro & 98,90 & 99,95 & 99,97 & 99,95 & 99,97 \\
\cline { 2 - 7 } & Dez & 99,85 & 99,92 & 99,92 & 99,97 & 99,97 \\
\hline \multirow{3}{*}{ Elevação de tensão } & Um & 97,22 & 97,89 & 98,25 & 97,67 & 97,60 \\
\cline { 2 - 7 } & Quatro & 98,85 & 97,75 & 98,02 & 99,00 & 99,97 \\
\cline { 2 - 7 } & Dez & 98,92 & 98,27 & 98,42 & 98,65 & 99,80 \\
\hline \multirow{3}{*}{ Notch } & Um & 99,90 & 99,95 & 99,95 & 99,97 & 99,97 \\
\cline { 2 - 7 } & Quatro & 99,95 & 99,95 & 99,95 & 99,95 & 99,97 \\
\cline { 2 - 7 } & Dez & 99,95 & 99,95 & 99,95 & 99,95 & 99,97 \\
\hline \multirow{3}{*}{ Sub-harmônico } & Um & 92,75 & 91,12 & 92,15 & 90,35 & 99,92 \\
\cline { 2 - 7 } & Quatro & 97,97 & 99,02 & 99,32 & 98,95 & 99,32 \\
\cline { 2 - 6 } & Dez & 99,90 & 99,95 & 99,95 & 99,97 & 99,85 \\
\hline
\end{tabular}

Fonte: Os autores (2020).

\subsection{Classificação de Distúrbios}

Neste item contem a análise de classificação para as seis classes apresentadas (sinal sem distúrbio e cinco classes de distúrbios). Considera-se, inicialmente, o distúrbio spike, por esse se manifestar de forma periódica no sinal monitorado e ocorrer em regime permanente no sistema elétrico. Em seguida, foi inserido um distúrbio por vez, simulando como o fluxo de dados ocorreria na prática, aonde todos os distúrbios considerados foram apresentados, no decorrer do tempo, ao sistema de detecção/classificação.

O algoritmo evolutivo proposto neste trabalho realizou a detecção e a classificação dos distúrbios de maneira recursiva e online. Esses processos acontecem ao mesmo tempo, ou seja, os distúrbios foram sempre apresentados com o sinal sem distúrbio ao algoritmo evolutivo que realizou a detecção e classificação dos mesmos. Portanto, se uma nova amostra é suficientemente diferente do conhecimento atual do sistema, então, cria-se uma nova regra só para representá-la em princípio; se não, adapta-se uma regra no sentido de incluir a amostra e reconhecer amostras semelhantes que aparecerem no futuro. Assim, o modelo vai evoluindo e adaptando-se ao longo do tempo percebendo (detectando) novos distúrbios e classificando-os nas seis classes consideradas.

As medidas de erro RMSE (Root Mean Squared Error) e NDEI (Non Dimensional Error Index) foram utilizadas para avaliar a precisão dos modelos. O RMSE é definido como a raiz quadrada do erro quadrático médio. O NDEI é a razão entre o RMSE e o desvio padrão da saída desejada. A seguir tem-se a formulação matemática do RMSE e NDEI, respectivamente: 


$$
\begin{gathered}
R M S E=\frac{1}{K} \sum_{k=1}^{k_{c}} \sqrt{\left({ }^{\prime} y_{(k+1)}-y_{k+1}\right)^{2}}, \\
N D E I=\frac{R M S E}{\sigma\left(y_{(k) \forall k}\right)},
\end{gathered}
$$

em que $k_{c}$ é o número de iterações, ' $y_{(k+1)}$ é a saída desejada do classificador, $y_{(k+1)}$ é a saída estimada do classificador, e $\sigma\left(y_{(k)} \forall k\right)$ é o desvio padrão da saída desejada

Após a inserção dos cinco distúrbios considerados neste trabalho, FBeM identificou seis classes (cinco distúrbios mais o sinal sem distúrbio). A Tabela 2 apresenta o desempenho (Acc) de classificação do modelo FBeM, o tempo computacional requerido (em segundos) pelo modelo, o RMSE e o NDEI.

\begin{tabular}{|c|c|c|c|c|c|}
\hline Distúrbios & Dados & $\operatorname{Acc}(\%)$ & Tempo(s) & RMSE & NDEI \\
\hline \multirow{15}{*}{$\begin{array}{c}\text { Spike, } \\
\text { Notch, } \\
\text { Sub-harmônico, } \\
\text { Transitório } \\
\text { oscilatório } \\
\text { e } \\
\text { Elevação de tensão, }\end{array}$} & $20 \mathrm{~dB}$ _1 ciclo & 80,15 & 45,58 & 0,2799 & 0,8397 \\
\hline & 20 dB_4ciclos & 89,95 & 33,84 & 0,1809 & 0,5427 \\
\hline & $20 \mathrm{~dB} \_10$ ciclos & 89,64 & 35,15 & 0,1583 & 0,4750 \\
\hline & $30 \mathrm{~dB} \_1$ ciclo & 92,75 & 37,78 & 0,1420 & 0,4260 \\
\hline & $30 \mathrm{~dB}$ _4ciclos & 97,19 & 30,93 & 0,0814 & 0,2442 \\
\hline & $30 \mathrm{~dB} \_10$ ciclos & 98,12 & 27,78 & 0,0625 & 0,1874 \\
\hline & 40 dB_1ciclo & 92,62 & 37,79 & 0,1317 & 0,3950 \\
\hline & $40 \mathrm{~dB} \_4$ ciclos & 98,81 & 29,60 & 0,0541 & 0,1624 \\
\hline & $40 \mathrm{~dB} \_10$ ciclos & 98,62 & 30,86 & 0,0560 & 0,1681 \\
\hline & 50 dB_1 ciclo & 93,30 & 36,74 & 0,1261 & 0,3782 \\
\hline & $50 \mathrm{~dB} \_4$ ciclos & 98,62 & 31,04 & 0,0557 & 0,1672 \\
\hline & $50 \mathrm{~dB} \_10$ ciclos & 99,07 & 29,44 & 0,0545 & 0,1595 \\
\hline & $60 \mathrm{~dB} \_1$ ciclo & 92,44 & 38,44 & 0,1352 & 0,4055 \\
\hline & $60 \mathrm{~dB} \_4$ ciclos & 98,38 & 30,31 & 0,0601 & 0,1802 \\
\hline & $60 \mathrm{~dB} \_10$ ciclos & 99,58 & 28,34 & 0,0519 & 0,1506 \\
\hline
\end{tabular}

Tabela 2 - Desempenho de classificação do modelo proposto, FBeM

Fonte: Os autores (2020).

Nota-se, que o modelo FBeM obteve desempenho bastante promissor, ou seja, acima de 98\%, conforme Ferreira (2010), comprovando dessa forma e eficiência dos sistemas de detecção/classificação e de extração de características. O modelo FBeM obteve o melhor resultado de classificação (acurácia e medida de erro) para $60 \mathrm{~dB}$ e dez ciclos.

\subsection{Resultados Comparativos entre Métodos}

Neste item o desempenho comparativo de classificação entre o modelo FBeM e os modelos MLR (Multiple Linear Regression), rede neural MLP (Multilayer Perceptron), e eTS (evolving Takagi-Sugeno) é apresentado. A comparação é realizada considerando a mesma base de dados utilizada no modelo FBeM, alterando-se somente o classificador. 
A Regressão Linear Múltipla é uma coleção de técnicas estatísticas para construir modelos que descrevem de maneira razoável relações entre várias variáveis explicativas de um determinado processo (GUARNIERI, 2006). A rede neural do tipo MLP é uma rede neural composta por vários neurônios do tipo perceptron. São redes multicamadas não recorrentes que utilizam um algoritmo de retropropagação do erro para ajuste dos pesos sinápticos, como, por exemplo, o backpropagation (BISHOP, 1995). Os sistemas fuzzy baseados em regras do tipo Takagi-Sugeno (TS), são sistemas nos quais os antecedentes das regras são termos linguísticos, e os consequentes são funções das variáveis antecedentes. O modelo Takagi-Sugeno evolutivo (eTS, evolving Takagi-Sugeno) propõe uma atualização do sistema de forma recursiva. Proposto por Angelov (2002), o modelo eTS determina os antecedentes das regras fuzzy por um processo de agrupamento não supervisionado, e os consequentes por meio de alguma variação de algoritmo de Quadrados Mínimos Recursivo.

A Tabela 3 apresenta o resultado comparativo entre os classificadores, considerando a acurácia.

Tabela 3 - Resultado comparativo de modelos em termos da acurácia na classificação

\begin{tabular}{c|c|c|c|c|c}
\hline Distúrbios & Dados & FBeM & eTS & MLP & MLR \\
\hline \multirow{5}{*}{$\begin{array}{c}\text { Spike, } \\
\text { Notch, }\end{array}$} & 20dB_1ciclo & 80,15 & 74,29 & 75,49 & 61,80 \\
\cline { 2 - 6 } & 20dB_4ciclos & 89,95 & 85,47 & 84,51 & 62,66 \\
\cline { 2 - 6 } Sub-harmônico, & 20dB_10ciclos & 89,64 & 86,00 & 87,89 & 62,51 \\
\cline { 2 - 6 } $\begin{array}{c}\text { Transitório } \\
\text { oscilatório }\end{array}$ & 30dB_1ciclo & 92,75 & 86,10 & 89,31 & 60,86 \\
\cline { 2 - 6 } e & 30dB_4ciclos & 97,19 & 98,11 & 95,11 & 62,11 \\
\cline { 2 - 6 } & 30dB_10ciclos & 98,12 & 98,00 & 97,03 & 62,19 \\
\cline { 2 - 6 } Elevação de tensão & 40dB_1ciclo & 92,62 & 91,40 & 90,25 & 60,18 \\
\cline { 2 - 6 } & 40dB_4ciclos & 98,81 & 94,48 & 95,78 & 62,00 \\
\cline { 2 - 6 } & 40dB_10ciclos & 98,62 & 98,55 & 97,88 & 63,66 \\
\cline { 2 - 6 } & 50dB_1ciclo & 93,30 & 92,04 & 90,05 & 61,08 \\
\cline { 2 - 6 } & 50dB_4ciclos & 98,62 & 98,48 & 96,70 & 61,78 \\
\cline { 2 - 6 } & 50dB_10ciclos & 99,07 & 98,34 & 97,89 & 63,44 \\
\cline { 2 - 6 } & 60dB_1ciclo & 92,44 & 92,22 & 90,67 & 60,55 \\
\cline { 2 - 6 } & 60dB_4ciclos & 98,38 & 97,41 & 96,19 & 62,40 \\
\cline { 2 - 6 } & 60dB_10ciclos & 99,58 & 98,57 & 98,09 & 63,80 \\
\hline
\end{tabular}

Fonte: Os autores (2020).

Percebe-se pela Tabela 3 que o modelo FBeM obteve desempenho acima dos outros modelos utilizados na comparação. Em seguida vem o modelo eTS, a rede neural MLP e a Regressão Linear Múltipla. Esses resultados demonstram a grande capacidade dos modelos evolutivos, especialmente o FBeM, no agrupamento de dados de forma incremental e através de um fluxo contínuo de dados, obtendo excelentes resultados na detecção e classificação de distúrbios de qualidade da energia elétrica em modo online e em tempo real, mesmo comparando com modelos offline (MLP e MLR). 


\subsection{Matriz de Confusão}

A matriz de confusão oferece uma medida efetiva do modelo de classificação, ao mostrar o número de classificações corretas versus as classificações erradas para cada classe, sobre um conjunto de $N$ exemplos. Cada coluna da matriz representa as amostras de uma classe prevista, enquanto as linhas representam os casos de uma classe estimada pelo modelo. As entradas da matriz são representadas por $M\left(C_{i}, C_{j}\right)$ indicando o número de exemplos de $N$ que são da classe $C_{i}$, mas que foram classificados pela hipótese $h$ como sendo da classe $C_{j}$. O número de acertos se localiza na diagonal principal $M\left(C_{i}, C_{i}\right)$ da matriz; os demais elementos $M\left(C_{i}, C_{j}\right)$, para $i \neq j$, representam erros na classificação.

$$
M\left(C_{i}, C_{j}\right)=\sum_{\left\{\forall(x, y) \in N: y=C_{i}\right\}}\left\|h(x)=C_{j}\right\|
$$

A matriz de confusão de um classificador ideal possui todos os elementos fora da diagonal iguais a zero, uma vez que ele não comete erros. A matriz de confusão possibilita analisar a porcentagem de assiduidade das diversas amostras associadas a uma classe, assim como permite o entendimento e a quantificação das amostras classificadas de forma errada. A matriz de confusão foi utilizada para avaliar o modelo FBeM quanto ao erro (confusão) na classificação entre as diversas classes e para verificar possibilidades que otimizem sua saída. As classes são distribuídas da seguinte forma: Classe 1: distúrbio spike; Classe 2: distúrbio notch; Classe 3: distúrbio sub-harmônico; Classe 4: distúrbio transitório oscilatório; Classe 5: distúrbio elevação de tensão e Classe 6: sinal sem distúrbio.

A Quadro 1 apresenta a matriz de confusão (melhor desempenho) para a relação sinalruído de $60 \mathrm{~dB}$ e 10 ciclos de análise da senoide do modelo FBeM. Percebe-se pela Quadro 1 como cada amostra foi classificada (acertos e erros). Os acertos estão na diagonal principal da matriz. Os erros estão identificados pelos dados localizados fora da matriz principal. As classes 1, 2, 4 e 6 foram as que obtiveram melhores resultados, com desempenho de 100\% (colunas saídas desejadas). Esse desempenho é de fundamental relevância para a classe 6 (sinal sem distúrbio), pois significa que o modelo é capaz de identificar quando o sinal não tem distúrbio de QEE. Na classe 5 (elevação de tensão) tem-se o pior desempenho com 28 amostras classificadas erradas. Dessas, 10 amostras foram classificadas como sinal sem distúrbio e 18 amostras classificadas como sub-harmônico. Em seguida tem-se a classe 3 (sub-harmônico) com 22 erros de classificação. Apresentou-se 8 amostras classificadas com sinal sem distúrbio e 14 amostras classificadas como elevação de tensão. O modelo apresentou desempenho total de 99,58\%, com 51 amostras classificadas erradas em um universo de 12000 amostras. 


\section{Quadro 1 - Matriz de confusão de classes obtida a partir do uso do modelo FBeM}

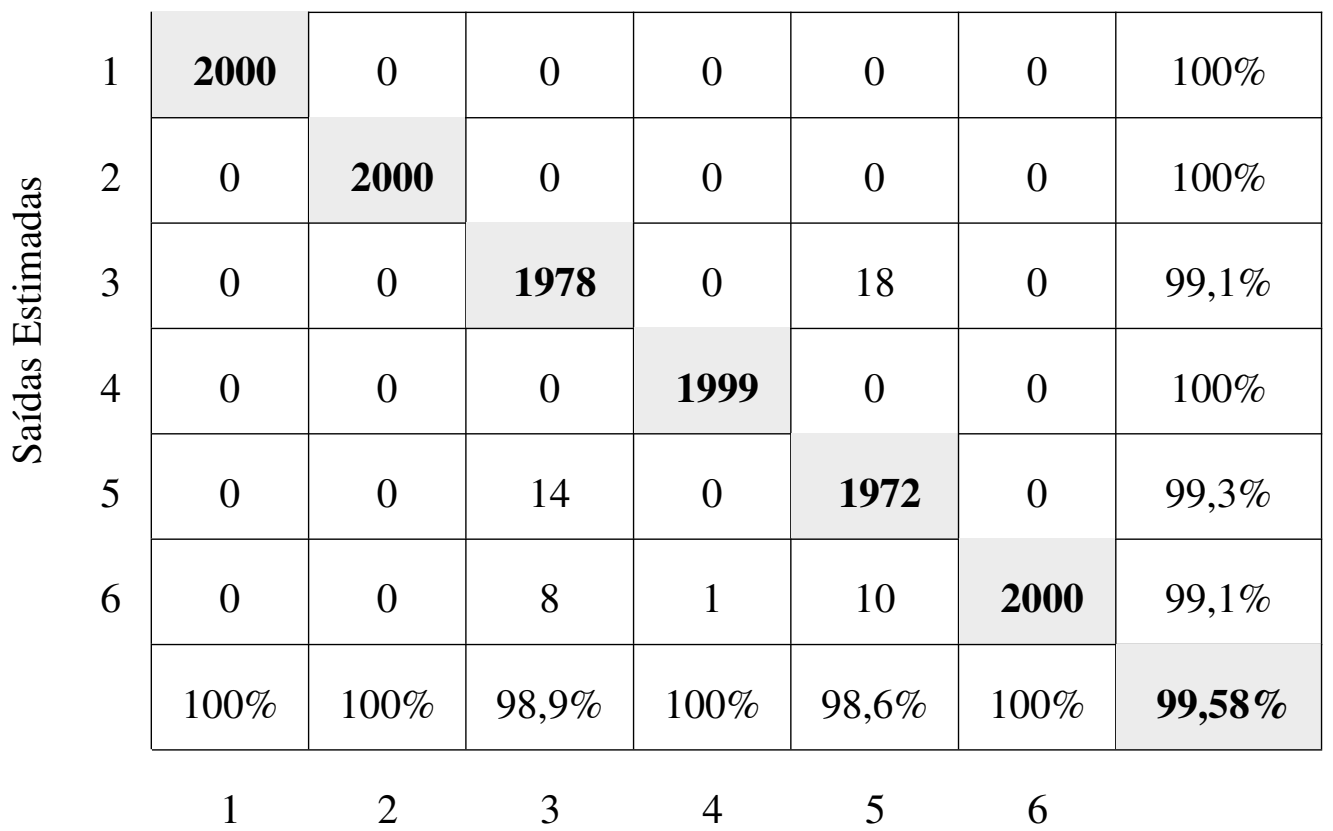

Saídas Desejadas

Fonte: Os autores (2020).

\section{CONCLUSÃO}

Os modelos evolutivos são bastante eficientes em problemas de classificação em tempo real, onde os dados são fornecidos na forma de fluxo. Em cada instante, tanto a estrutura quanto os parâmetros que descrevem o algoritmo são atualizados em tempo real. Nese contexto, este trabalho propôs a modelagem evolutiva baseada em conjuntos fuzzy (FBeM), para a detecção e classificação de distúrbios de QEE. FBeM emprega grânulos de informação tipo fuzzy para construir mapas granulares que de maneira geral associam dados granulares de entrada a dados granulares de saída.

O desempenho de classificação foi promissor, mesmo considerando baixos níveis de relação sinal-ruído, poucos ciclos de análise da senoide e várias classes de distúrbios consideradas ao mesmo tempo, demonstrando, assim, a robustez do modelo em ambientes dinâmicos e sujeito a novidades. Em comparação com outros modelos de classificadores, FBeM mostrou-se superior tanto para o melhor caso (10 ciclos e $60 \mathrm{~dB}$ ) com acurácia em 99,58\% quanto para o caso mais difícil ( 1 ciclo e $20 \mathrm{~dB}$ ) com acurácia em 80,15\%, apresentando alto desempenho, ou seja, poucos erros de classificação e com baixo custo computacional (tempo de processamento pequeno).

Para trabalhos futuros propõe-se gerar uma maior variedade de amostras que representam as classes de distúrbio e considerar outros tipos de distúrbios de qualidade da energia elétrica. Além disso, propõe-se, também, considerar menor número de ciclos de senoides por janela, para a extração de características. A formulação mais complicada do problema naturalmente levará à necessidade de empregar métodos de aprendizado não supervisionados e 
incrementais mais elaborados, e utilização de novas técnicas de extração de características. Em especial, abordaremos metodologias de seleção incremental de atributos para tornar o sistema evolutivo ainda mais flexível. 


\section{REFERÊNCIAS}

ANGELOV, P. Evolving rule-based models: a tool for design of flexible adaptive systems. London: Springer-Verlag, 2002.92 p.

BISHOP, C. Neural networks for pattern recognition. New York: Oxford University Press, 1995.

BISOI, R.; DASH, P. K.; DAS, P. P. Short-term electricity price forecasting and classification in smart grids using optimized multikernel extreme learning machine. Neural Computing and Applications, v. 32, n. 5, p. 1457-1480, 2020.

DI SANTO, K. G. et al. A review on smart grids and experiences in brazil. Renewable and Sustainable Energy Reviews, London, v. 52, p. 1072-1082, Dec 2015.

DUGAN, R. C. Electrical power systems quality. 2. ed. New York: McGraw-Hill, 2003. 521 p.

EL-HAWARY, M. E. The smart grid: state-of-the-art and future trends. Electric Power Systems Research, UK, v. 42, n. 3/4, p. 42-239, Feb 2014.

FERREIRA, D. Análise de distúrbios elétricos em sistemas de potência. 2010. 233 p. Tese (Doutorado em Engenharia Elétrica) - Universidade Federal do Rio de Janeiro, Rio de Janeiro, 2010.

GARCIA, C. et al. Evolvable fuzzy systems from data streams with missing values: With application to temporal pattern recognition and cryptocurrency prediction. Pattern Recognition Letters, v. 128, p. 278-282, 2019.

GUARNIERI, R.A. Emprego de redes neurais artificiais e regressão linear múltipla no refinamento das previsões de radiação solar do modelo Eta. 2006. Master's thesis Instituto Nacional de Pesquisas Espaciais, São José dos Campos, 2006.

HODRICK, R.; PRESCOTT, E.C. Postwar u.s. business cycles: an empirical investigation. Journal of Money, Ohio, v. 29, n. 1, p. 1-16, Feb 1997.

LATHI, B. P.; GREEN, R. A. Linear systems and signals. New York: Oxford University Press, 2005.

LEITE, D.; COSTA, P.; GOMIDE, F. Evolving granular classification neural networks. In: IEEE INTERNATIONAL JOINT CONFERENCE ON NEURAL NETWORKS. Atlanta: [s.n.], 2009. p. 1736-1743.

LEITE, D.; GOMIDE, F. Evolving linguistic fuzzy models from data streams. In: Combining Experimentation and Theory. Berlin: Springer, 2012. p. 209-223.

LEITE, D. et al. Fuzzy granular neural network for incremental modeling of nonlinear chaotic systems. In: 2016 IEEE INTERNATIONAL CONFERENCE ON FUZZY SYSTEMS (FUZZ-IEEE). [S.1.]: IEEE, 2016. p. 64-71.

LEITE, D.; ŠKRJANC, I. Ensemble of evolving optimal granular experts, owa aggregation, and time series prediction. Information Sciences, v. 504, p. 95-112, 2019.

LIU, H. et al. Complex power quality disturbances classification via curvelet transform and deep learning. Electric Power Systems Research, v. 163, p. 1-9, 2018. 
OLIVEIRA, J. C. Qualidade da energia elétrica: definição e a nálise dos i tens de qualidade. Universidade Federal de Uberlândia, Uberlândia, p. 90, 2000.

SILVA, S. et al. Evolving neuro-fuzzy network for real-time high impedance fault detection and classification. Neural Computing and Applications, p. 1-14, 2018.

ŠKRJANC, I. et al. Evolving fuzzy and neuro-fuzzy approaches in clustering, regression, identification, and classification: A survey. Information Sciences, v. 490, p. 344-368, 2019.

SOARES, E. et al. Evolving fuzzy set-based and cloud-based unsupervised classifiers for spam detection. IEEE Latin America Transactions, v. 17, n. 09, p. 1449-1457, 2019.

STIEGLER, S. M. A modest proposal: a new standard for the normal. The American Statistician, Washington, v. 36, n. 2, p. 137-138, Mar 1982.

THE INSTITUTE OF ELECTRICAL AND ELECTRONICS ENGINEERS. IEEE recommended practice for monitoring electric power quality. IEEE, New York, p. 91, 2009.

TOMIC, J.; KUSLJEVIC, M.; VUJICIC, V. A new power system digital harmonic analyzer. IEEE Transactions on Power Delivery, New York, v. 22, n. 2, p. 772-780, Apr 2007.

WANG, D.; PEDRYCZ, W.; LI, Z. Granular data aggregation: an adaptive principle of the justifiable granularity approach. IEEE Transactions on Cybernetics, v. 49, n. 2, p. 417-426, 2018.

YAGER, R. R. Measures of specificity over continuous spaces under similarity relations. Fuzzy Sets and Systems, New Rochelle, v. 159, n. 17, p. 2193-2210, Sept 2008.

ZHU, R. et al. Power quality disturbances classification via fully-convolutional siamese network and k-nearest neighbor. Energies, v. 12, n. 24, p. 4732, 2019. 